\title{
Video Article \\ Femtosecond Laser Filaments for Use in Sub-Diffraction-Limited Imaging and Remote Sensing
}

\author{
Matthews M. Springer ${ }^{1}$, Benjamin D. Strycker ${ }^{1,2}$, Kai Wang ${ }^{1}$, Alexei V. Sokolov ${ }^{1,2}$, Marlan O. Scully ${ }^{1,2}$ \\ ${ }^{1}$ Texas A\&M University \\ ${ }^{2}$ Baylor University
}

Correspondence to: Matthews M. Springer at matt.m.springer@gmail.com

URL: https://www.jove.com/video/58207

DOI: doi: $10.3791 / 58207$

Keywords: Engineering, Issue 146, Femtosecond filaments, remote sensing, ultrafast spectroscopy, sub-diffraction-limited imaging, nonlinear optics, laser-induced-breakdown spectroscopy

Date Published: 4/25/2019

Citation: Springer, M.M., Strycker, B.D., Wang, K., Sokolov, A.V., Scully, M.O. Femtosecond Laser Filaments for Use in Sub-Diffraction-Limited Imaging and Remote Sensing. J. Vis. Exp. (146), e58207, doi:10.3791/58207 (2019).

\section{Abstract}

Probing remote matter with laser light is a ubiquitous technique used in circumstances as diverse as laser-induced breakdown spectroscopy and barcode scanners. In classical optics, the intensity that can be brought to bear on a remote target is limited by the spot size of the laser at the distance of the target. This spot size has a lower bound determined by the diffraction limit of classical optics. However, amplified femtosecond laser pulses generate intensity sufficient to modify the refractive index of the ambient air and undergo self-focusing. This self-focusing effect leads to the generation of highly intense laser filaments which maintain their intensity and small sub-millimeter diameter size at distances well beyond the classical Rayleigh length. Such intensity provides the capability of remote scanning, imaging, sensing, and spectroscopy with enhanced spatial resolution. We describe a technique for generating filaments with a femtosecond regenerative chirped-pulse amplifier, and for using the resulting filament to conduct imaging and spectroscopic measurements at remote distances of at least several meters.

\section{Video Link}

The video component of this article can be found at https://www.jove.com/video/58207/

\section{Introduction}

The spatial coherence and corresponding small divergence angle of laser beams have led to numerous applications in remote sensing, including chemically-sensitive measurements of the atmosphere ${ }^{1,2}$, range-finding ${ }^{3}$, and remote spectroscopy ${ }^{4}$. The same coherence properties allow very tight focusing of laser light that can deliver continuous focused intensities of billions of watts per square centimeter and pulsed intensities of $10^{13}$ watts per square centimeter over a period of a few femtoseconds. Such extreme intensities are useful for numerous applications including examining the nonlinear optical properties of matter $^{5}$, precision optical micromachining ${ }^{6}$, materials characterization through laser-inducedbreakdown spectroscopy ${ }^{7}$, stimulated Raman spectroscopy ${ }^{8,9,10}$, and trace chemical detection ${ }^{11}$.

However, the physical limitations of Gaussian beams set limits on the ability to apply these properties of extreme intensity and small divergence angle simultaneously. A laser beam focused to a small spot size will necessarily diverge with a greater angle. Classically, the beam divergence angle is given by, where $\lambda$ is the wavelength and $w_{0}$ is the radius of the beam waist. Since the divergence angle is set by the diameter of the laser beam and the focal length $f$ of the focusing lens, , and tight focusing is not possible at distances of many meters as $f$ becomes large compared to $d$.

Workers in the field of amplified femtosecond pulses noticed that this limitation on intensity vs. range was violated for high-intensity femtosecond pulses, with burn marks smaller than the diffraction limit appearing on targets at large distance from the originating laser ${ }^{12}$. This was found to be due to Kerr-effect self-focusing. The refractive index of the air is modified in proportion to the intensity of the laser field, and when the laser has a Gaussian intensity profile, the resulting refractive intensity profile becomes functionally a lens ${ }^{5}$. The beam self-focuses as it propagates, resulting in a narrow and intense filament of less than $100 \mu \mathrm{m}$ radius whose small size is maintained by a dynamic balance among classical diffraction, Kerr self-focusing, and defocusing due to plasma generation ${ }^{13}$.

With femtosecond laser filaments, intensities on the order of $10^{13} \mathrm{~W} / \mathrm{cm}^{2}$ can be delivered to targets at distances of many meters with commercially-available femtosecond chirped-pulse amplifiers. Thus, many experiments which previously required tight focusing conditions and targets very close to a lens of high numerical aperture can now be done at distance more typical of remote sensing applications. However, intensities much higher than this threshold are not easily possible with filamentation, as the beam tends to break up into multiple filaments where each individual filament is near the critical power for self-focusing ${ }^{13}$.

Numerous applications are possible. We present a protocol primarily applicable to imaging and spectroscopy of remote targets using a femtosecond laser filament scanned over the target surface. The experimental setup is shown in Figure 1. 


\section{Creation of the Femtosecond Laser Filament}

1. As femtosecond filaments require the output of a Class 4 laser, wear appropriate eye protection rated for the particular laser system in use and establish a clear and well-defined beam line with an appropriate beam dump. Follow all standard laser safety procedures.

2. Begin with the output of a pulsed, amplified femtosecond laser whose instantaneous output power is greater than or equal to the critical power for self-focusing in air, about 3.2 GW for a Ti:Sapphire laser at $800 \mathrm{~nm}$ wavelength. Generate the amplified pulse in a commercial femtosecond laser amplifier system using manufacturer's protocol. In practice, pulse energy of about $1 \mathrm{~mJ}$ for an approximately $35 \mathrm{fs}$ pulse is sufficient. Good results are obtained with pulse energy of 2-4 mJ.

3. Pass the laser beam through an iris that slightly clips the outer edges. It is observed to promote filament formation, since filament formation is known to be seeded by sharp gradients and inhomogeneity in the spatial intensity profile of the laser.

4. Pass the beam through the converging lens that has a focal length of approximately $200 \mathrm{~cm}$ or greater, so that the geometric focusing is not so great that self-focusing is overwhelmed by optical breakdown or diffraction. Slightly tilt the lens with respect to the direction of propagation, since additional anisotropy is known to help seed the self-focusing process.

5. Observe a filament at a location near the geometric focus of the lens. Diagnose filamentation by a diffuse (several-mm-sized) halo surrounding a bright (approximately 100- $\mu \mathrm{m}$-sized) core. The halo could be seen on a white paper and the bright cores usually flicker.

1. Additionally, observe a characteristic self-phase modulation process in the air, which produces bright, multi-colored conical emission rings that are visible beyond the filament. For lasers with energies which are several times the threshold for filamentation, multiple filaments are observed. These are visible as multiple bright spots in the conical emission pattern, and can be eliminated by attenuation before the iris.

\section{Remote Scanning of the Target Surface}

1. Put a two-axis motorized translation stage capable of moving the sample in the direction transverse to the propagation of the laser beam on the table. Ensure that the laser beam is incident on the center of the stage. Bolt the stage on the table with screws. For laboratory purposes, it is generally easier to keep the laser beam fixed in space while scanning the target under the beam.

2. Place sand in a container $(5 \mathrm{~mm} \times 25.4 \mathrm{~mm} \times 25.4 \mathrm{~mm})$. The thickness of sand is around $2 \mathrm{~mm}$.

1. Put the metals (copper, stainless steel, aluminum) on the top of sand (Figure 3a). Cover the metals with another $2 \mathrm{~mm}$ layer of sand (Figure 3b).

2. With the laser off, put the container in the center of the translation stage. Make sure that the center of container is at the location where filamentation is observed for step 1.1-1.5.

3. Set up the laser's computer control to fire a single shot when electronically commanded. Write a LabVIEW or a similar computer language to perform the control. For automated single-shot pulses, an external trigger is required.

1. Connect a trigger TTL pulse to the External Trigger port on the back of the laser control module with a BNC cable. Enable the external trigger option on the laser control module. The TTL pulse will now trigger the laser to fire a single shot.

4. Set up the appropriate sensor apparatus. Set up the entrance of the spectrometer pointing to the impact point.

1. Use a lens to couple the light from filamentation impact point into a spectrometer. Make sure that the distance between the lens and filamentation is about the focal length.

2. Connect the spectrometer with computer using USB cable. Use software to monitor the spectrum. Open the software and the spectrum, and then click the Run button.

3. Use the mouse to zoom in the range that is recorded in the experiment. Optimize the spectrometer position after seeing the signal on the screen.

4. For imaging measurements, replace the spectrometer with a photomultiplier tube or a CCD camera.

5. Write a program in LabVIEW or a similar computer language to perform a loop over the following steps: Fire a single shot from the laser; collect and save the resulting data; move the translation stage to the next coordinate point.

\section{Representative Results}

The resolution of the scanned images is limited optically only by the $\sim 100 \mu \mathrm{m}$. Therefore, the translation stage motion should be of this order of magnitude or smaller for maximum resolution. However, this level of resolution is not necessary for all measurements. This protocol has been used for both imaging ${ }^{14}$ and spectroscopic ${ }^{15}$ measurements. Figure 1 shows the experimental setup. The pulse is generated in an amplifier system. The pulse is $1 \mathrm{kHz}, 50 \mathrm{fs}$, and centered at $800 \mathrm{~nm}$. Figure 2 compares a scan of a small Texas A\&M logo target taken with a laser at the diffraction limit compared to a scan taken with a filament-forming beam. This experiment was performed using filaments in liquid water, but the results may be rescaled for air in remote sensing ${ }^{13}$. Figure 3 shows spatially-resolved filament-induced breakdown spectroscopy scans of metal objects of different composition buried approximately two millimeters below a layer of sand. The shapes and compositions of the metal objects are apparent. In general, filamentation provides a number of mechanisms for target effects. The initial pulse can provide information on the surface layer, while subsequent pulses can provide information on deeper portions of the material through ablation or mechanical removal of surface layers. 


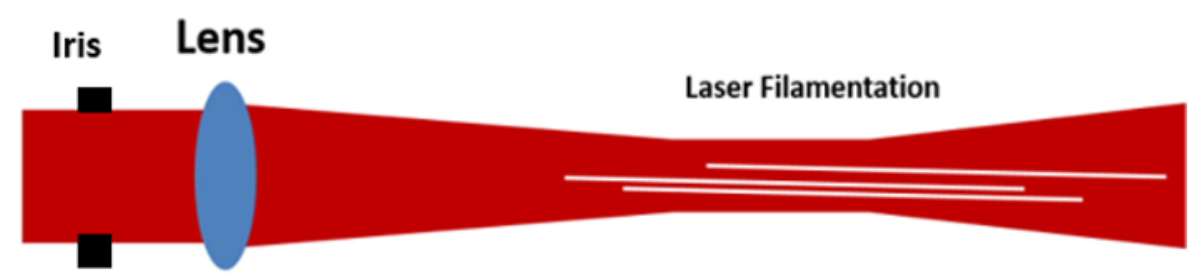

$800 \mathrm{~nm}, 50 \mathrm{fs}, 1 \mathrm{KHz}$

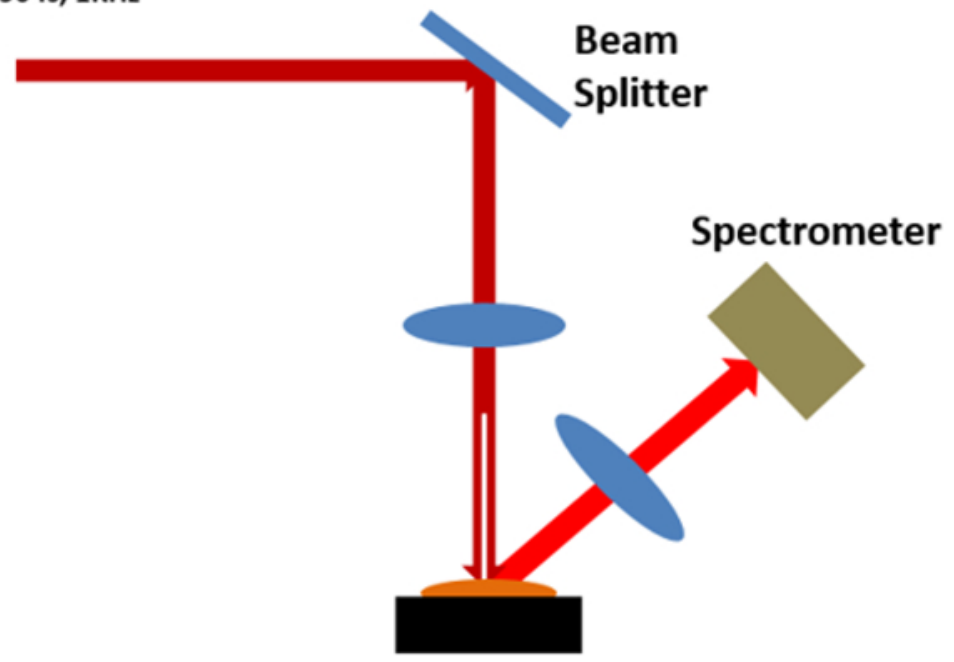

\section{Translation Stage}

Figure 1. The experimental setup. The laser is $1 \mathrm{kHz}, 50 \mathrm{fs}$, and centered at $800 \mathrm{~nm}$. It is focused with a lens to reach the intensity $\left(\sim 10^{13} \mathrm{~W} /\right.$ $\mathrm{cm}^{2}$ ) to form laser filaments. The object is under sand and put on a translation stage. The scattered light is collected with a spectrometer. Please click here to view a larger version of this figure.

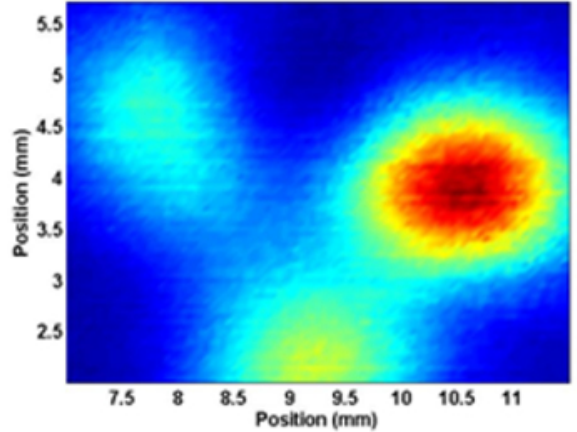

(a)

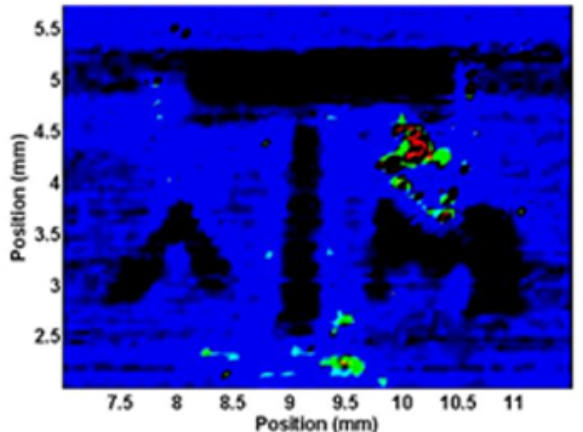

(b)

Figure 2. Sub-diffraction-limited Imaging. Remote images generated by scanning a laser beam across a printed Texas A\&M logo at a distance of several meters. a) Logo imaged with non-filamented beam. b) Logo imaged with filamented beam. Please click here to view a larger version of this figure. 

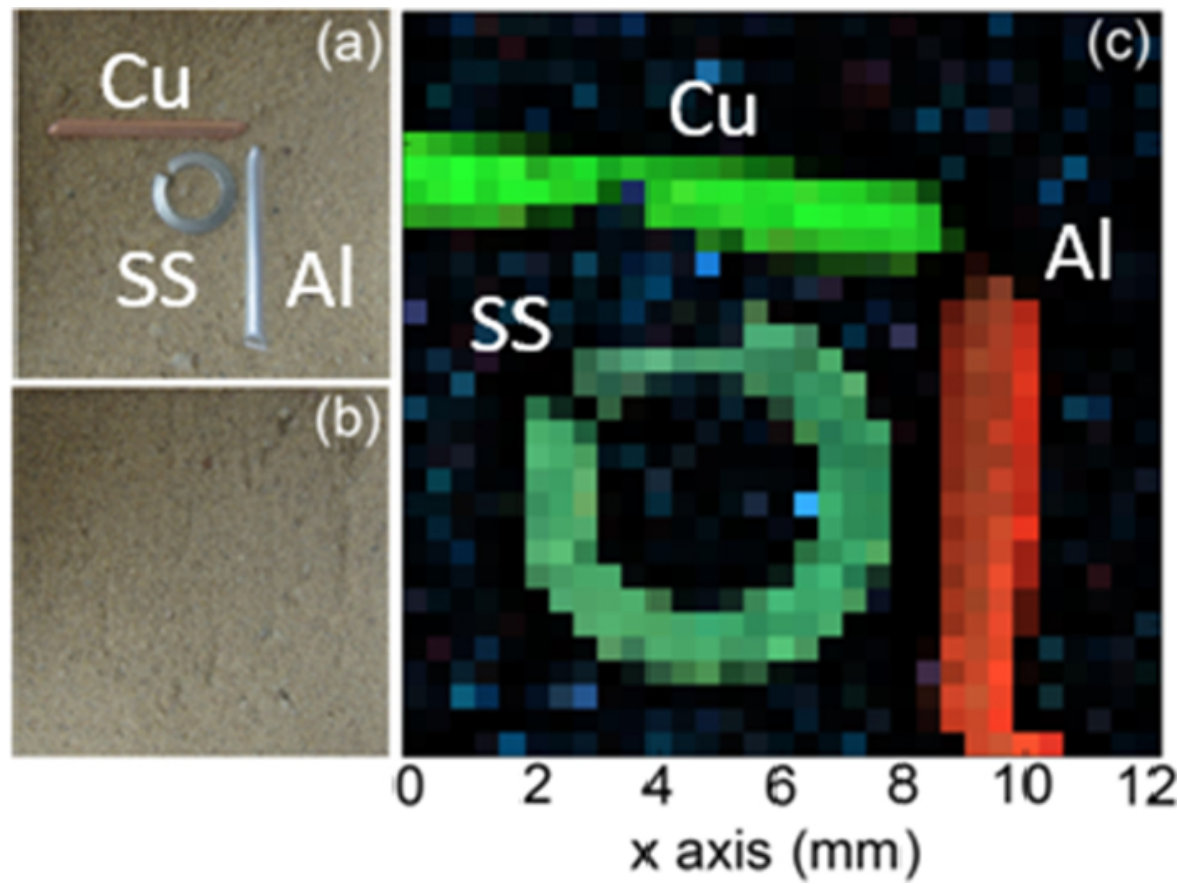

Figure 3. The chemical map. Spectrally and spatially resolved image of metal objects buried under sand. a) Objects above sand. b) Objects below $2.3 \pm 0.3 \mathrm{~mm}$ of sand. c) Image with material composition color-coded to metal spectral features. Composite image of the buried objects with aluminum (Al), copper (Cu), and stainless steel (SS) corresponding to the red, green, and cyan color components, respectively Please click here to view a larger version of this figure.

\section{Discussion}

The method presented above is a laboratory protocol for the use of high-intensity laser light delivered at classically intractable distances. Of the numerous possible applications of such light - CARS, FIBS, THz radiation, photoacoustics, superradiance, etc. - many applications can deliver point information about surface material properties. Femtosecond laser filaments with sub-classical-diffraction-limited spot size allows use of these techniques while scanning the surface on a point-by-point basis. This protocol is an ideal test bed for the development of such techniques.

The most critical aspect of the protocol is to generate the laser filamentation. To generate the stable laser filamentation, the critical laser intensity is a few $10^{13} \mathrm{~W} / \mathrm{cm}^{2}$ and the clamped intensity is around $1.4 \times 10^{14} \mathrm{~W} / \mathrm{cm}^{2}$ measured in experiment ${ }^{16}$. There is no laser filamentation when the intensity is either high or low. If the intensity is too high, the medium might be ionized strongly at the focal point and a laser induced break-down will happen. A bright spark instead of a laser filamentation will be observed. In that case, attenuate the power or use a lens with a longer focal length. Conversely, if the power is low (no plasma generation is observed), increase the power or use a lens with short focal length. Moreover, in either case, it is worthwhile to adjust the chirp to help to form a laser filamentation.

This scanning technique is generally better suited for laboratory use and proof-of-concept rather than field deployment since remote sensing in the field generally does not allow fine translation-stage control of the target under investigation. In those scenarios the same lab-developed laser techniques can be used, but the laser itself will have to be scanned through more traditional beam steering methods such as changing the orientation of the laser apparatus itself.

The protocol could be relatively easily extended to involve experiments with multiple filaments, filament bundles, pump-probe experiments, standoff spectroscopy, waveguide, or numerous other possibilities. In each case, one of the major experimental hurdles is the alignment of the intersecting focal spots, but with this protocol, this need only be done once. The optical elements are fixed in place and the sample itself is the only object required to move. This can be done very precisely with a translation stage. Further modification of this protocol to achieve further control over the location of the filament formation distance, including filament formation at hundreds of meters from the laser, is possible in principle by careful control of the output laser pulse. Multi-filamentation will also form a waveguide during the propagation, which could help to deliver a light in free space.

Remote sensing is a broad subject that spans disciplines such as physics, chemistry, engineering, environmental science, etc. In the supplementary material, we propose additional remote sensing schemes including stand-off spectroscopy and superradiance in addition to filamentation.

\section{Disclosures}

No conflicts of interest declared. 


\section{Acknowledgments}

The research is supported by the Office of Naval Research (ONR) (Award N00014-16-1-2578 and N00014-16-1-3054), Robert A. Welch Foundation (Grant No. A-1547, No. A-1261), Air Force Office of Scientific Research (Award No. FA9550-18-1-0141), SMART Fellowship and a grant from King Abdulaziz City for Science and Technology (KACST).

\section{References}

1. Kocharocsky, V., et al. Gain-swept superrandiance applied to the stand-off detection of trace impurities in the atmosphere. Proceedings of the National Academy of Sciences of the United States of America. 102 (22), 7806-7811 (2005).

2. Hemmer, P., et al. Standoff spectroscopy via remote generation of a backward-propagation laser beam, Proceedings of the National Academy of Sciences of the United States of America. 108 (8) 3130-3134 (2011).

3. Zuber, M.T., et al. The Mars Observer laser altimeter investigation, Journal of Geophysical Research. 97 ( E5), 7781 (1992).

4. Mejean, G., et al. Remote detection and identification of biological aerosols using a femtosecond terawatt lidar system. Applied Physics $B$ : Lasers and Optics. 78 (5), 535-537 (2004).

5. Boyd, R.W., Nonlinear Optics. Academic Press (2008).

6. Gattass, R.R., and Mazur, E. Femtosecond laser micromachining in transparent materials, Nature Photonics. 2, $219-225$ (2008).

7. Tognoni, E., Palleschi, V., Corsi, M., and Christoforetti, G. Quantitative micro-analysis by laser-induced breakdown spectroscopy: a review of the experimental approaches. Spectrochimica Acta Part B: Atomic Spectroscopy. 57 (7), 1115-1130 (2002).

8. Beadie, G., et al. Toward a FAST CARS anthrax detector: coherence preparation using simultaneous femtosecond laser pulses. Optics Communications. 244, 423-430 (2005).

9. Scully, M.O., et al. FAST CARS: Engineering of a laser spectroscopic technique for rapid identification of bacterial spores. Proceedings of the National Academy of Sciences of the United States of America. 99 (17), 10994-11001 (2002).

10. Pestov, D., et al. Optimizing the laser-pulse configuration for coherent Raman spectroscopy. Science. 316 (5822), 265-268 (2007).

11. Braun, A. Self-channeling of high-peak-power femtosecond laser pulses in air. Optics Letters. 20 (1), 73-75 (1995).

12. Couairon, A. and Mysyrowicz, A. Femtosecond filamentation in transparent media. Physics Reports. 441, 47-189 (2007).

13. Wang, K., et al. Remote sub-diffraction imaging with femtosecond laser filaments. Optics Letters. 37 (8), 1343-1345 (2012).

14. Strycker, B.D., Wang, K., Springer, M.D., Sokolov, A.V. Chemical-specific imaging of shallowly buried objects using femtosecond laser pulses. Applied Optics. 52 (20), 4792-4796 (2013).

15. Heck, G., Sloss, J., Levis, R.J. Adaptive control of the spatial position of white light filaments in an aqueous solution. Optics Communications. 259 (1), 216-222 (2006).

16. Li, H.L. et al. Critical power and clamping intensity inside a filament in a flame. Optics Express. 24 (4), 3424 (2016). 